\title{
INVESTIGAÇÃO DO POTENCIAL ANTIOXIDANTE E ANTICOLINESTERÁSICO DE Hippeastrum (AMARYLLIDACEAE)
}

\author{
Raquel Brandt Giordani, Letícia Balvedi Pagliosa, Amélia T. Henriques e José Angelo S. Zuanazzi* \\ Faculdade de Farmácia, Universidade Federal do Rio Grande do Sul, Av. Ipiranga, 2752, 90610-000 Porto Alegre - RS, Brasil \\ Julie H. A. Dutilh \\ Departamento de Botânica, Instituto de Botânica, Universidade Estadual de Campinas, 13083-970 Campinas - SP, Brasil
}

Recebido em 20/12/07; aceito em 4/6/08; publicado na web em 5/11/08

\begin{abstract}
ANTIOXIDANT AND ANTICOLINESTERASIC EFFECTS OF Hippeastrum SPECIES (AMARYLLIDACEAE). Amaryllidaceae family is an important source of bioactive molecules and considering the taxonomic relationships, it is relevant to investigate the genus Hippeastrum. Thus, the aim of this work was to determine antioxidant and anticholinesterasic activities of Hippeastrum morelianum, Hippeastrum psittacinum and Hippeastrum santacatarina. Both antioxidant and acetylcholinesterasic activities of extracts were determinated by bioautography as $10 \mathrm{mg} / \mathrm{mL}$ and $0.1 \mathrm{mg} / \mathrm{mL}$, respectively. The antioxidant activity of alkaloid lycorine, determinated spectrophotometrically with $\mathrm{DPPH}$, indicated an $\mathrm{IC}_{50}$ value of $0.326 \mathrm{mM}$.
\end{abstract}

Keywords: Amaryllidaceae; antioxidant; anticholinesterasic.

\section{INTRODUÇÃO}

A etiologia da Doença de Alzheimer (DA) permanece desconhecida, mas estudos post mortem têm mostrado que a doença é caracterizada pela diminuição da função colinérgica no cérebro. ${ }^{1}$ Os mais modernos medicamentos utilizados para tratar os sintomas da DA elevam os níveis de acetilcolina pela inibição da enzima acetilcolinesterase (AChE). Ensaios desenvolvidos para detectar esta atividade estão sendo amplamente utilizados para avaliar um grande número de plantas utilizadas popularmente para melhorar a memória. ${ }^{2}$

Sabe-se também que o dano causado por espécies reativas de oxigênio, atualmente, é considerado um fator contribuinte de diversas doenças, incluindo a DA. ${ }^{3}$ Assim, identificar o potencial antioxidante de uma substância pode credenciá-la a ser uma molécula bioativa mais completa quando se considera o conjunto de suas propriedades. Dessa forma, é adequada a associação de ambos os ensaios para um estudo bioguiado mais eficiente.

$\mathrm{Na}$ busca por novos fármacos, os produtos naturais destacam-se pela diversidade estrutural e, assim, as plantas são candidatas importantes para screening de novos compostos bioativos. Ao considerar as informações botânicas e quimio-taxonômicas, a eleição das plantas a serem investigadas torna-se mais selecionada. Considerando esse conjunto de fatores, a família Amaryllidaceae, particularmente, desponta como uma fonte promissora de novas substâncias bioativas, justificando a avaliação dos gêneros presentes no Brasil.

O alcalóide galantamina foi, inicialmente, isolado de Galanthus woronowii, mas atualmente é obtido a partir de Narcissus sp. e Leucojum aestivum, assim como sinteticamente. A partir da descoberta deste alcalóide enquanto potente inibidor da enzima $\mathrm{AChE}$, sua aprovação pelo FDA (Food and Drug Administration) em 2001 para comercialização e, conseqüentemente, importante utilização na DA, o interesse no estudo químico e farmacológico da família Amaryllidaceae vem aumentando exponencialmente. ${ }^{4,5}$ Um screening do extrato bruto e das frações enriquecidas em alcalóides obtidas durante a extração ácido-base auxilia no direcionamento das investigações de isolamento e elucidação estrutural dessas substâncias, bem como contribui para

*e-mail: zuanazzi@farmacia.ufrgs.br a triagem de gêneros e espécies promissoras.

$\mathrm{O}$ gênero Hippeastrum compreende aproximadamente 50 espécies, todas originárias da América do Sul, sendo que destas, 30 ocorrem no Brasil. ${ }^{6}$ Poucos estudos botânicos, químicos e farmacológicos são encontrados na literatura para o gênero Hippeastrum em comparação aos demais gêneros de ocorrência na Europa e Ásia.

Nosso grupo tem investigado sistematicamente a composição química e atividades farmacológicas de plantas do gênero Hippeastrum do Brasil, sendo os resultados obtidos bastante promissores. ${ }^{7}$ O presente trabalho teve como objetivo realizar um screening das atividades antioxidante, pelo método do DPPH, e anticolinesterásica, pelo ensaio autobiográfico, dos extratos de três espécies: Hippeastrum santacatarina (Traub) Dutilh, Hippeastrum morelianum Lem. e Hippeastrum psittacinum Herb., bem como de um alcalóide isolado.

\section{PARTE EXPERIMENTAL}

\section{Procedimentos experimentais gerais}

Todos os solventes e reagentes utilizados apresentavam grau analítico adequado. O radical DPPH (2,2-difenil-1-picril-hidrazila) foi adquirido da Aldrich Co, assim como a enzima acetilcolinesterase $500 \mathrm{U}$ e fisostigmina. Rutina e boldina foram adquiridos da Merck. Análise por cromatografia líquida de alta eficiência (CLAE) foi desenvolvida em equipamento Alliance 2695 (Waters) e detector Waters 996 (UV-diodos em série). A coluna cromatográfica utilizada foi Luna Phenomenex -C18 (75 x 4,6 mm), com sistema eluente já otimizado para análise de extratos de Amaryllidaceae. ${ }^{7}$

\section{Material vegetal}

Raízes, partes aéreas e bulbos de Hippeastrum morelianum Lem. (UCE 14351) e bulbos de Hippeastrum psittacinum Herb. (UCE 143513) foram coletados na serra de Atibaia - SP; bulbos de Hippeastrum santacatarina (Traub) Dutilh (ICN 149429) foram coletados no município de São Francisco de Paula-RS. As exsicatas encontram-se depositadas nos herbários da Universidade Estadual de Campinas (UCE) e Universidade Federal do Rio Grande do Sul (ICN). 


\section{Preparação dos extratos}

Para as três espécies investigadas, o procedimento de obtenção dos extratos foi padronizado. $\mathrm{O}$ material vegetal fresco foi triturado, separadamente, sendo cada parte (raiz, bulbo e partes aéreas) submetida à maceração em etanol comercial $\left(96^{\circ} \mathrm{GL}\right)$, durante $24 \mathrm{~h}$. Após maceração e filtração, o solvente foi eliminado em evaporador rotatório e a água residual do vegetal eliminada por liofilização, resultando um extrato bruto dessecado.

O marco vegetal foi recolocado em maceração, repetindo-se o processo descrito anteriormente até reação negativa frente ao Reativo de Dragendorff, ${ }^{8}$ obtendo-se, assim, um extrato bruto (EB).

Esse extrato (EB) foi submetido a fracionamento ácido-básico para alcalóides, modificado, conforme demonstrado na Figura 1.

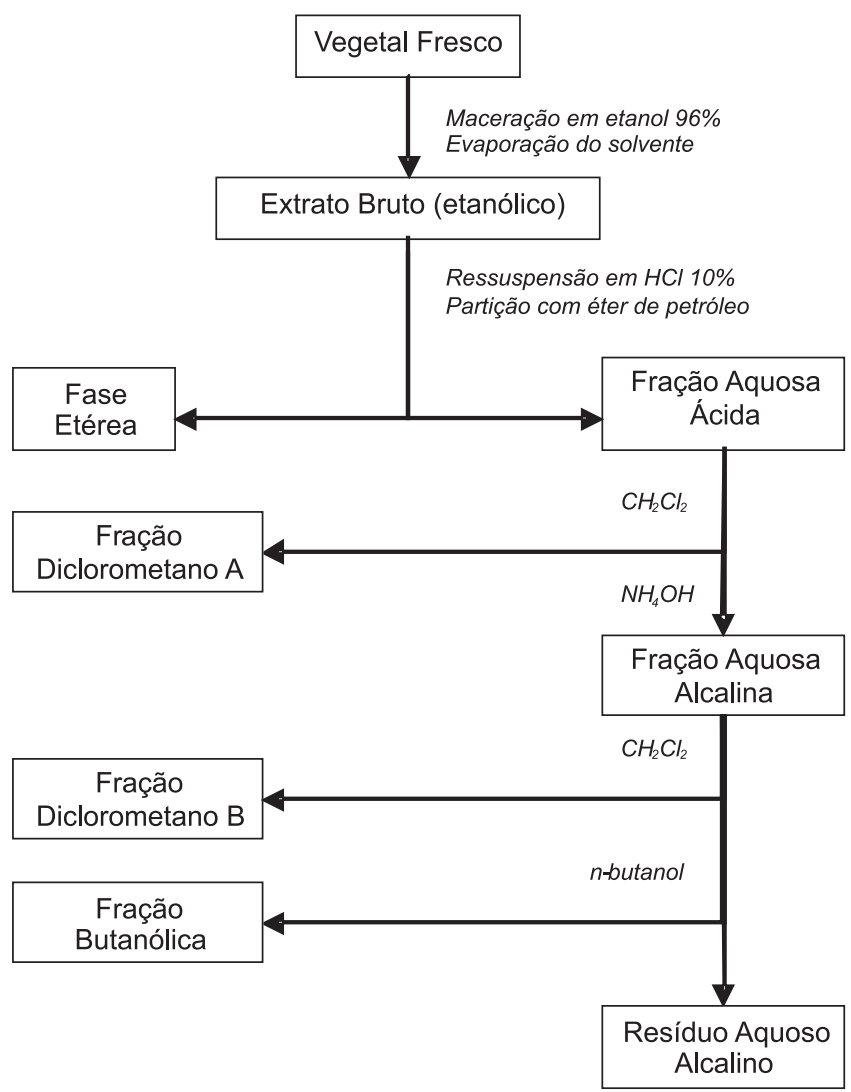

Figura 1. Fluxograma do procedimento de extração utilizado para obtenção dos extratos

Durante o procedimento de alcalinização do extrato de $H$. santacatarina um sólido amorfo precipitou, em grande quantidade. Com auxílio de métodos cromatográficos, espectroscópicos e dados disponíveis na literatura essa substância foi caracterizada como o alcalóide licorina (Figura 2), comum na família Amaryllidaceae. Esse alcalóide apresenta ampla gama de atividades biológicas já relatadas, tais como antitumoral, ${ }^{9}$ indutora de apoptose, ${ }^{10}$ antiinflamatória ${ }^{11}$ e anticolinesterásica, ${ }^{12}$ entretanto não há relatos para a atividade antioxidante.

Análises por CLAE dos extratos e frações contemplados por este trabalho indicam que o alcalóide licorina está presente apenas em amostras de Hippeastrum santacatarina. A Figura 3 evidencia o perfil cromatográfico da fração Diclorometano B, onde a licorina é o componente mais abundante, embora também esteja presente no extrato bruto e frações Diclorometano A e $n$-butanólica.

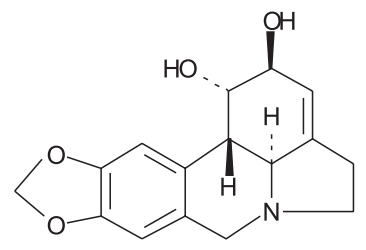

Figura 2. Estrutura do alcalóide licorina

\section{Preparação das amostras}

As amostras de extrato bruto, fração Diclorometano A, fração Diclorometano B e fração $n$-butanólica das três espécies, foram solubilizadas em etanol e posteriormente diluídas, caracterizando as seguintes concentrações: 10,1 e 0,1 mg/mL. A partir de cada diluição, alíquotas de $10 \mu \mathrm{L}$ foram aplicadas sobre as cromatoplacas dos testes antioxidante e anticolinesterásico. Para a avaliação da licorina, foi preparada uma solução estoque (em metanol) de $1 \mathrm{mg} / \mathrm{mL}(3,48 \mathrm{mM})$ da qual foram retiradas alíquotas de 5, 10 e $15 \mu \mathrm{L}$ para realização do teste qualitativo e demais alíquotas para o teste quantitativo.

\section{Análise qualitativa da atividade anticolinesterásica}

O método utilizado consistiu numa autobiografia desenvolvida sobre uma cromatoplaca de sílica gel, onde foram aplicadas as amostras. A enzima acetilcolinesterase (500 U) foi dissolvida em tampão Tris $0,05 \mathrm{M}(\mathrm{pH} 7,8)$ seguida da adição de $150 \mathrm{mg}$ de albumina de soro bovino para estabilização da mesma. Esta solução foi mantida à temperatura de $4{ }^{\circ} \mathrm{C}$ e nebulizada sobre a cromatoplaca com as amostras. A placa foi incubada a $37^{\circ} \mathrm{C}$, durante $20 \mathrm{~min}$.

A placa foi, em seguida, nebulizada com uma solução contendo 1-naftil acetato, em etanol, e Fast Blue B salt, em água, ocorrendo o desenvolvimento de coloração púrpura após 1 a 2 min. As regiões da placa que contêm substâncias capazes de inibir a ação da enzima acetilcolinesterase (neste caso ocorre formação do diazônio púrpura) apresentam uma mancha branca contra a coloração de fundo. ${ }^{13}$ Como padrão positivo foram utilizados $5 \mu \mathrm{L}$ de uma solução-mãe a $1 \mathrm{mg}$ / $\mathrm{mL}(3,63 \mathrm{mM})$ do alcalóide fisostigmina.

\section{Análise qualitativa da atividade antioxidante}

Sobre uma cromatoplaca de sílica gel foram aplicadas, pontualmente, as amostras testadas. Após a total evaporação do veículo de solubilização de amostra, a placa foi nebulizada com solução metanólica de DPPH a 0,2 mM. A atividade antioxidante foi eviden-

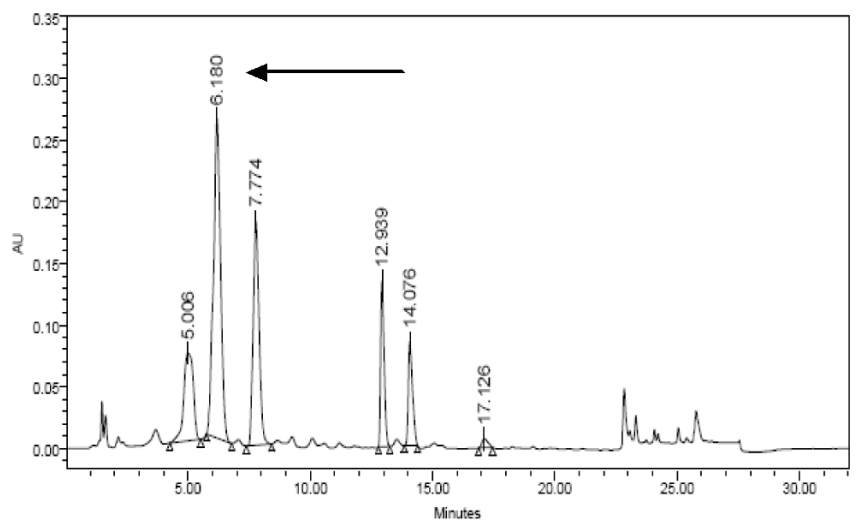

Figura 3. Cromatograma da fração Diclorometano $B$, dos bulbos de $H$. santacatarina, evidenciando a presença do alcalóide licorina (indicado pela seta) 
ciada pela presença de manchas brancas ou amarelas decorrentes da redução do DPPH, contra a coloração púrpura de fundo, após 30 min à temperatura ambiente. ${ }^{14}$ Como padrão positivo foram utilizados 5 $\mu \mathrm{L}$ de solução-mãe a $1 \mathrm{mg} / \mathrm{mL}(1,64 \mathrm{mM})$ de rutina.

\section{Análise quantitativa da atividade antioxidante}

O composto isolado, por precipitação, durante a extração ácidobase, foi avaliado também frente ao DPPH, utilizando-se espectrofotômetro UV/VIS. ${ }^{15}$ Primeiramente, foram preparados $10 \mathrm{~mL}$ de solução estoque de DPPH em metanol na concentração de aproximadamente $0,1 \mathrm{mM}$, mantida sob refrigeração e protegida da luz. A partir dela foram feitas diluições até 0,$087 ; 0,075 ; 0,062 ; 0,050 ; 0,037 ; 0,025$; 0,0125 e $0,0025 \mathrm{mM}$. A curva de calibração foi construída a partir dos valores de absorvância a $517 \mathrm{~nm}$ das soluções, tendo o metanol como referência, em triplicata. A equação da curva de calibração do DPPH foi $y=9,3981 x-0,0092\left(R^{2}=0,993\right)$, onde y corresponde à concentração de DPPH no meio $(\mathrm{mM})$ e x à absorvância.

O equipamento foi zerado com metanol e a absorção da solução metanólica de DPPH $(0,1 \mathrm{mM})$, assim como das amostras de licorina $(0,069 ; 0,139 ; 0,278 ; 0,348 ; 0,383 ; 0,389$ e $0,400 \mathrm{mM})$, foi medida a $517 \mathrm{~nm}$. Para tanto, foi preparada uma solução metanólica de amostra com concentração final de $3,48 \mathrm{mM}$, da qual foram retiradas alíquotas para obtenção da concentração final desejada. O alcalóide boldina foi utilizado como padrão positivo na concentração de $0,075 \mathrm{mM}$. Um $\mathrm{mL}$ da amostra foi adicionado de $1 \mathrm{~mL}$ da solução metanólica de DPPH, à temperatura ambiente, e após 30 min procedeu-se à leitura em espectrofotômetro $(517 \mathrm{~nm})$. Por ação de um antioxidante (AH) o DPPH' é reduzido, formando difenil-picril-hidrazina, de coloração amarela, com conseqüente desaparecimento da absorção, podendo a mesma ser monitorada pelo decréscimo da absorvância.

A partir da equação da curva de calibração e dos valores de absorvância para cada concentração testada, foram determinados os percentuais de DPPH remanescentes $\left(\% \mathrm{DPPH}_{\mathrm{REM}}\right)$, conforme a Equação 1:

$\% \mathrm{DPPH}_{\mathrm{REM}}=[\mathrm{DPPH}]_{\text {amostra }} /[\mathrm{DPPH}]_{\text {inicial }} \mathrm{x} 100$

onde $[\mathrm{DPPH}]_{\text {amostra }}$ corresponde à concentração de DPPH no meio após a reação com a amostra e $[\mathrm{DPPH}]_{\text {inicial }}$ é a concentração inicial de DPPH $(0,1 \mathrm{mM}){ }^{17,18}$

A porcentagem de atividade antioxidante (\%AA) corresponde à quantidade de $\mathrm{DPPH}^{*}$ consumida, sendo que a quantidade de amostra necessária para decrescer a concentração inicial de DPPH em $50 \%$ é denominada de concentração inibitória $\left(\mathrm{CI}_{50}\right)$. Os valores de absorvância em todas as concentrações testadas foram convertidos em \%AA pela Equação 2:

$\% \mathrm{AA}=\left\{\left[\mathrm{Abs}_{\text {controle }}-\left(\mathrm{Abs}_{\text {amostra }}-\mathrm{Abs}_{\text {branco }}\right)\right] \times 100\right\} / \mathrm{Abs}_{\text {controle }}$

onde $\mathrm{Abs}_{\text {controle }}$ é a absorvância inicial da solução metanólica de DPPH e Abs ${ }_{\text {amostra }}$ é a absorvância da mistura reacional (DPPH + amostra). ${ }^{18}$

\section{Análise estatística}

Os resultados apresentados neste estudo correspondem à média de três repetições $(n=3) \pm$ desvio padrão da média. Foram considerados estatisticamente diferentes os resultados de atividade antioxidante que apresentaram probabilidade de ocorrência de hipótese de nulidade menor que 5\% $(\rho<0,05)$ aplicando-se ANOVA, seguido de comparações múltiplas pelo teste de Tukey. $\mathrm{O}$ valor de $\mathrm{CI}_{50}$ foi calculado com auxílio do software GraphPad.

\section{RESULTADOS E DISCUSSÃO}

O estudo com plantas monocotiledôneas encontra, de início, uma grande dificuldade de identificação botânica. Para a família Amaryllidaceae, particularmente para o gênero Hippeastrum, não é diferente. Alguns gêneros dessa família, especialmente aqueles de ocorrência na Europa, vêm sendo estudados há muitos anos com expressivos resultados botânicos, químicos e farmacológicos e, comparativamente, o gênero Hippeastrum mostra-se ainda em um estágio inicial de investigação. Suas características químicas estão sendo delineadas, assim como seu perfil botânico e caracterização de suas espécies.

A necessidade de tornar mais objetivas e menos dispendiosas as pesquisas por constituintes químicos de plantas levou ao desenvolvimento de numerosas técnicas de ensaios para monitoramento e seleção de extratos e substâncias bio/farmacologicamente úteis. ${ }^{18}$ Dentre eles, quanto à busca de inibidores da AChE, destacam-se dois testes autobiográficos, ${ }^{13,17}$ dos quais o método de Marston, utilizado neste trabalho, apresenta melhor limite de detecção.

O teste com DPPH é um método bastante conveniente para o screening antioxidante de pequenas moléculas, uma vez que a reação pode ser observada visualmente utilizando-se uma cromatoplaca ou espectrofotômetro UV/VIS. ${ }^{16}$

A análise qualitativa da atividade anticolinesterásica revelou que, de acordo com o método empregado, todas as amostras testadas, de $H$. morelianum, $H$. psittacinum e $H$. santacatarina, apresentaram resultado positivo ao teste. As amostras extrato bruto e Diclorometano B de $H$. santacatarina $(10 ; 1 ; 0,1 \mathrm{mg} / \mathrm{mL})$ apresentaram intensidade de descoloração semelhante ao padrão fisostigmina $(1 \mathrm{mg} / \mathrm{mL})$.

Dentre os resultados obtidos após avaliação qualitativa pelo método do DPPH, os extratos brutos EB dos bulbos de $H$. morelianum, $H$. psittacinum e $H$. santacatarina $(10 \mathrm{mg} / \mathrm{mL})$ apresentaram atividade similar ao padrão positivo rutina $(1 \mathrm{mg} / \mathrm{mL})$. Para as demais amostras, os resultados variaram de acordo com a espécie testada e estão descritos na Tabela 1, a qual sumariza os resultados qualitativos obtidos.

Em ambos os ensaios autobiográficos qualitativos, antioxidante e anticolinesterásico, a licorina apresentou resultado positivo nas mesmas concentrações que os padrões positivos. Assim, o teste quantitativo, pelo método do DPPH, tornou-se uma ferramenta para determinar a $\mathrm{CI}_{50}$ dessa substância para a atividade antioxidante. $\mathrm{O}$ resultado verificado para o potencial anticolinesterásico da licorina está em acordo com os dados da literatura para essa substância.

Os resultados obtidos corroboram a afirmativa de que exemplares da família Amaryllidaceae são fontes potenciais de compostos anticolinesterásicos. No entanto, investigações para isolamento e elucidação estrutural dos seus alcalóides são necessárias e justificadas a partir de todas as frações oriundas da extração ácido-base. A reação de descoloração da fração Diclorometano B semelhante à do padrão positivo, neste ensaio, pode ser atribuída à presença majoritária de alcalóides nessa amostra, a qual está sendo investigada em estudo bioguiado.

Verifica-se, a partir dos testes qualitativos, que os resultados dos ensaios antioxidantes empregados não mostraram relação direta com os resultados anticolinesterásicos. Entretanto, é necessária a utilização de diferentes métodos de avaliação para uma definição desta atividade para este grupo químico.

O alcalóide licorina foi submetido à avaliação quantitativa da atividade antioxidante frente ao DPPH pelo método fotocolorimétrico. As concentrações testadas foram baseadas nos resultados da autobiografia.

A Figura 4 apresenta a curva de calibração construída para o DPPH, a qual forneceu a equação para cálculo dos resultados das amostras. Por sua vez, a Figura 5 sumariza os resultados obtidos, após 
Tabela 1. Potencial antioxidante frente ao DPPH e screening da atividade acetilcolinesterásica das amostras de $H$. morelianum, H. santacatarina e $H$. psittacinum

\begin{tabular}{|c|c|c|c|c|c|c|c|c|c|c|}
\hline & \multicolumn{2}{|c|}{$\begin{array}{l}\text { RAÍZES } \\
\text { H. morelianum }\end{array}$} & \multicolumn{2}{|c|}{$\begin{array}{l}\text { PARTES AÉREAS } \\
\text { H. morelianum }\end{array}$} & \multicolumn{2}{|c|}{$\begin{array}{c}\text { BULBOS } \\
\text { H. morelianum }\end{array}$} & \multicolumn{2}{|c|}{$\begin{array}{c}\text { BULBOS } \\
\text { H. santacatarina }\end{array}$} & \multicolumn{2}{|c|}{$\begin{array}{c}\text { BULBOS } \\
\text { H. psittacinum }\end{array}$} \\
\hline & DPPH & $\mathrm{AChE}$ & DPPH & AChE & DPPH & $\mathrm{AChE}$ & DPPH & AChE & DPPH & $\mathrm{AChE}$ \\
\hline \multicolumn{11}{|c|}{ EXTRATO BRUTO } \\
\hline $10 \mathrm{mg} / \mathrm{mL}$ & +++ & ++ & +++ & ++ & +++ & ++ & - & +++ & +++ & + \\
\hline $1 \mathrm{mg} / \mathrm{mL}$ & + & + & + & + & + & + & - & +++ & + & + \\
\hline $0,1 \mathrm{mg} / \mathrm{mL}$ & - & + & - & + & - & + & - & +++ & + & + \\
\hline \multicolumn{11}{|c|}{ DICLOROMETANO B } \\
\hline $10 \mathrm{mg} / \mathrm{mL}$ & + & ++ & +++ & + & +++ & +++ & +++ & +++ & ++ & +++ \\
\hline $1 \mathrm{mg} / \mathrm{mL}$ & - & ++ & + & + & + & ++ & ++ & +++ & - & + \\
\hline $0,1 \mathrm{mg} / \mathrm{mL}$ & - & + & - & + & - & + & + & +++ & - & - \\
\hline \multicolumn{11}{|c|}{ DICLOROMETANO A } \\
\hline $10 \mathrm{mg} / \mathrm{mL}$ & +++ & ++ & +++ & + & + & ++ & +++ & ++ & $\#$ & $\#$ \\
\hline $1 \mathrm{mg} / \mathrm{mL}$ & + & + & + & + & - & + & + & + & $\#$ & \# \\
\hline $0,1 \mathrm{mg} / \mathrm{mL}$ & - & + & - & + & - & + & + & - & $\#$ & \# \\
\hline \multicolumn{11}{|c|}{ FRAÇÃO BUTANÓLICA } \\
\hline $10 \mathrm{mg} / \mathrm{mL}$ & + & ++ & + & + & +++ & +++ & +++ & ++ & + & +++ \\
\hline $1 \mathrm{mg} / \mathrm{mL}$ & - & ++ & - & + & + & +++ & ++ & ++ & - & ++ \\
\hline $0,1 \mathrm{mg} / \mathrm{mL}$ & - & + & - & + & + & ++ & + & + & - & - \\
\hline
\end{tabular}

* Os resultados estão indicados em relação ao observado para os padrões: rutina (ensaio antioxidante) e fisostigmina (ensaio anticolinesterásico).

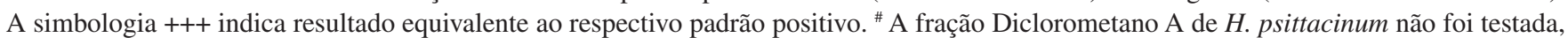
uma vez que apresentou baixo rendimento.

30 min de reação e leitura em espectrofotômetro, para as concentrações testadas de licorina, as quais foram comparadas e demonstraram diferença estatística significante. Ainda, verificou-se um padrão de atividade antioxidante não linear em relação à concentração da licorina, expressivo em concentrações de 0,348 a $0,400 \mathrm{mM}$. Para o padrão positivo, o alcalóide boldina, os resultados sugerem um efeito tempo dependente a 0,075 mM, sendo verificado após $1 \mathrm{~h}$ decréscimo de, aproximadamente, $100 \%$ na absorvância do DPPH (dados não demonstrados). De acordo com a literatura, a boldina apresenta relação linear entre concentração e atividade seqüestradora do radical livre DPPH de 0 a $0,300 \mathrm{mM}$, com valor de $\mathrm{CI}_{50}$ de $0,0552 \mathrm{mM}^{19}$

Considerando o potencial antioxidante da licorina em distintas concentrações, após 30 min de ensaio, foi determinado o $\mathrm{CI}_{50}$ deste alcalóide, com auxílio do software GraphPad, plotando-se na abscissa as concentrações da amostra $(\mathrm{mM})$ e na ordenada a porcentagem de inibição de DPPH. Assim, obteve-se uma curva cuja forma se assemelha a um comportamento sigmóide. A Figura 6 apresenta esta curva de concentração versus porcentagem de inibição do DPPH pela licorina, após $30 \mathrm{~min}$, cujo $\mathrm{CI}_{50}$ verificado foi $0,326 \mathrm{mM}$.

A partir dos resultados deste trabalho, estudos futuros poderiam

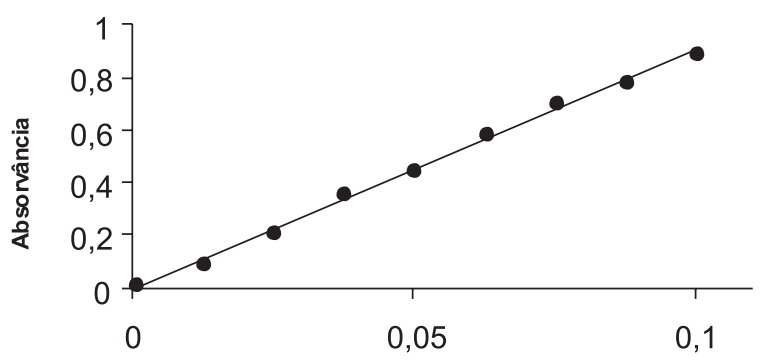

Concentração DPPH (mM)

Figura 4. Curva de calibração para o DPPH, em espectrofotômetro a 517 $n m$

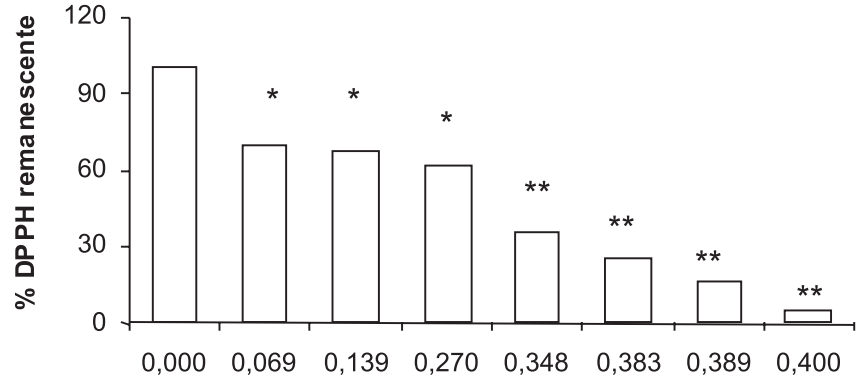

Concentração de Licorina $(\mathrm{mM})$

Figura 5. Atividade antioxidante do alcalóide licorina demonstrada pela diminuição da concentração de DPPH no meio à medida que aumenta a concentração de amostra, onde: *Diferença estatística significativa $(\rho<0,05)$ em relação à ausência de amostra ${ }^{* *}$ Diferença estatística significativa $(\rho<0,05)$ em relação à ausência de amostra e às concentrações 0,069; 0,139 e 0,270 mM

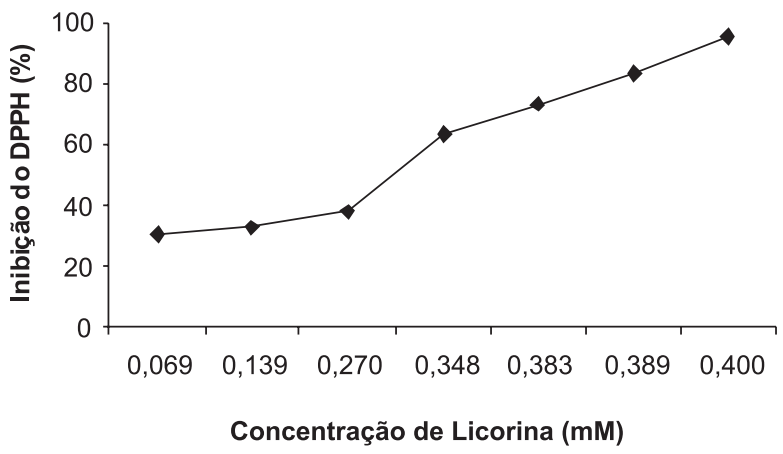

Figura 6. Representação da curva de concentração da licorina versus porcentagem de inibição do radical DPPH: comportamento não linear e $\mathrm{CI}_{50}$ $=0,326 \mathrm{mM}$ 
ser realizados visando o aprimoramento e exploração da atividade antioxidante da licorina testando-se derivados sintéticos, tais como acetatos e cloridratos.

\section{AGRADECIMENTOS}

Este trabalho contou com apoio financeiro da Coordenação de Aperfeiçoamento de Pessoal de Nível Superior (CAPES) e Fundação de Amparo à Pesquisa do Estado do Rio Grande do Sul (FAPERGS) e Bolsa do Conselho Nacional de Desenvolvimento Científico e Tecnológico (CNPq). Os autores agradecem à Profa. Dra. L. Eggers pela contribuição na identificação das espécies.

\section{REFERÊNCIAS}

1. Perry, N. S. L.; Bollen, C.; Perry, E. K.; Ballard, C.; Pharmacol. Biochem. Behav. 2003, 75, 651.

2. Houghton, P. J.; Ren, Y. Em Research in Amaryllidaceae; Govil, S. ed.; Studium Press: Houston, 2006, cap. 2.

3. Houghton, P. J.; Howes, M. J.; Lee, C. C.; Steventon, G.; J. Ethnopharmacol. 2007, 110, 391.

4. Elgorashi, E. E.; Stafford, G. I.; Van Staden, J.; Planta Med. 2004, 70, 260.

5. Martin, S. F. Em The Alkaloids: Chemistry and Physiology; Brossi, A. ed.; Academic Press Inc.: San Diego:, 1987, cap. 4.; Lewis, J. R.; Nat. Prod. Rep. 1990, 7, 549; Gabrielsen, B.; Monath, T. P.; Huggins, J. W.; Kefauver, D. F.; Pettit, G. R.; Groszek, G.; Hollingshead, M.; Kirsi, J. J.; Shannon, W. M.; Shubert, E. M.; Dare, J.; Ugarkar, B.; Ussery, M. A.; Phelan, M. J.; J. Nat. Prod. 1992, 55, 1569; Likhitwitayawuid, K.; Angerhofer, C. K.; Chai, H.; Pezzuto, J. M.; Cordell, G. A.; Ruangrungsi, N.; J. Nat. Prod. 1993, 56, 1331; Campbell, F. G.; Chambers, T. M.; Gammon, D. W.; Bastida, J.; Codina, C.; Viladomat, F.; Smith, P. J.; Albrecht, C. F.; Planta Med. 1998, 64, 91

6. Meerow, A. W.; Fay, M. F.; Guy, C. L.; Li, Q.; Zaman, F. Q.; Chase, M. W.; Am. J. Bot. 1999, 86, 1325.

7. Silva, A. F.; Andrade, J. P.; Bevilaqua, L. R.; Souza, M. M.; Izquierdo, I.; Henriques, A. T.; Zuanazzi, J. A.; Pharmacol. Biochem. Behav. 2006, 85,
148; Hofmann Jr, A. E.; Sebben, C.; Sobral, M.; Dutilh, J.; Henriques, A. T.; Zuanazzi, J. A.; Biochem. Syst. Ecol. 2003, 31, 1455; Castilhos, T.; Dissertação de Mestrado, Universidade Federal do Rio Grande do Sul, Brasil, 2004; Sebben, C.; Dissertação de Mestrado, Universidade Federal do Rio Grande do Sul, Brasil, 2005; Giordani, R. B.; Dissertação de Mestrado, Universidade Federal do Rio Grande do Sul, Brasil, 2007; Andrade, J. P.; Dissertação de Mestrado, Universidade Federal do Rio Grande do Sul, Brasil, 2007.

8. Bruneton, J.; Farmacognosia: Fitoquímica - Plantas Medicinales, $2^{\text {nd }}$ ed., Acribia S.A: Zaragoza, 2001.

9. Hua, D. H.; Saha, S.; Takemoto, D. J.; Anticancer Res. 1997, 17, 2435; Li, S.; Chen, C.; Zhang, H.; Guo, H.; Wang, H.; Wang, L.; Zhang, X.; Hua, S.; Yu, J.; Xiao, P.; Li, S.; Tan, X.; Antiviral Res. 2005, 67, 18; Liu, J.; Li, Y.; Tang, L. J.; Zhang, G. P.; Hu, W. X.; Biomed. Pharmacother. 2007, 61, 229; Hohmann, J.; Forgo, P.; Molnar, J.; Wolfard, K.; Molnar, A.; Thalhammer, T.; Mathe, I.; Sharpies, D.; Planta Med. 2002, 68, 454.

10. Li, Y.; Liu, J.; Tang, L.; Shi, Y.; Ren, W.; Hu, W.; Oncol. Rep. 2007, 17, 377.

11. Citoglu, G.; Tanker, M.; Gümüsel, B.; Phytot. Res. 1998, 12, 205.

12. Houghton, P. J.; Agbedahunsi, J. M.; Adegbulube, A.; Phytochemistry, 2004, 65, 2893; Shoei-Sheng, L.; Uppala, V.; Prasad, R. C.; Sio-Hong, L.; Jung-Hsin, L.; Bioorg. Med. Chem. 2007, 15, 1034; Lopez, S.; Bastida, J.; Viladomat, F.; Codina, C.; Life Sci. 2002, 71, 2521.

13. Marston, A.; Kissling, J.; Hostettmann, K.; Phytochem. Anal. 2002, 13, 51 .

14. Blois, M. S.; Nature. 1968, 181, 1199.

15. Shirwaikar, A.; Rajedran, K.; Punitha, I.; Biol. Pharmaceut. Bull. 2006, 29, 1906.

16. Sánchez-Moreno, C.; Larrauri, J. A.; Saura-Calixto, F.; J. Sci. Food. Agric. 1998, 76, 270; Brand-Williams, W.; Cuvelier, M. E.; Berset, C.; Lebensm.- Wiss. Technol. 1995, 28, 25.

17. Rhee, I. K.; Van der Meent, M.; Ingkaninan, K.; Verpoorte, R.; J. Chromatogr., A 2001, 915, 217.

18. Viegas Jr., C.; Bolzani, V. S.; Furlan, M.; Fraga, C. A. M.; Barreiro, E. J.; Quim. Nova. 2004, 27, 655.

19. Zhao, Q.; Zhao, Y.; Wang, K.; J. Ethnopharmacol. 2006, 106, 408. 